Service social

\title{
Why Men Are the Way they Are : the Male-Female Dynamic par Warren Farrell, New York, McGraw Hill, 1986, 403 pages.
}

\section{Thomas Antil}

Volume 37, numéro 1-2, 1988

Par-delà les barrières des sexes

URI : https://id.erudit.org/iderudit/706402ar

DOI : https://doi.org/10.7202/706402ar

Aller au sommaire du numéro

Éditeur(s)

École de service social de l'Université Laval

ISSN

1708-1734 (numérique)

Découvrir la revue

Citer ce compte rendu

Antil, T. (1988). Compte rendu de [Why Men Are the Way they Are : the Male-Female Dynamic par Warren Farrell, New York, McGraw Hill, 1986, 403 pages.] Service social, 37(1-2), 280-282. https://doi.org/10.7202/706402ar d'utilisation que vous pouvez consulter en ligne.

https://apropos.erudit.org/fr/usagers/politique-dutilisation/ 
En terminant, je citerai ce passage :

"Aussi, je ne soulignerai jamais assez combien nous devons être disponibles à l'égard de ces jeunes, ouverts à leur expression, attentifs à leurs besoins et à leurs émotions et respectueux de leur différence. Comprendre la vision que chaque jeune a de sa prostitution demeure indispensable à toute velléité d'aide. " (P. 123)

Guy Poulin

Groupe d'aide aux personnes impulsives (GAPI),

Québec.

Why Men Are the Way They Are : the Male-Female Dynamic par Warren FArRel, New York, McGraw Hill, 1986, 403 pages.

Warren Farrell s'est fait une réputation de pionnier en ce qui concerne la nouvelle condition masculine avec la publication, en 1974, de son premier livre, The Liberated Man. Depuis, il a organisé des centaines d'ateliers et de groupes de réflexion sur les rapports entre les hommes et les femmes. Dans ce dernier livre, Farrell répond aux questions les plus fréquemment posées lors de ses ateliers, par exemple: "Si les hommes ont le pouvoir, pourquoi voudraient-ils changer? " ou "Les femmes ont beaucoup changé, pourquoi les hommes n'ontils pas changé avec elles?". Les réponses apportées par Farrell risquent d'en surprendre ou d'en consterner plusieurs.

D'abord, l'auteur prétend qu'il n'est pas tout à fait vrai que les hommes ont plus de pouvoir que les femmes. Cette affirmation découle de la définition très large du pouvoir que Farrell présente au tout début de son livre : la capacité de contrôler sa propre vie dans toutes ses dimensions. Cela veut dire non seulement avoir accès aux récompenses extérieures (revenus, prestige, biens matériels), mais également aux ressources intérieures telles que la capacité d'exprimer ses émotions, une image positive de soi, une paix intérieure et une concordance entre ses valeurs et ses activités quotidiennes. S'il est vrai que les hommes ont traditionnellement eu beaucoup plus de pouvoir extérieur que les femmes, l'auteur constate que la compétition et les autres performances exigées par les rôles masculins les ont coupés du pouvoir sur leur vie intérieure; quand ils se mettent en contact avec leur expérience intime du pouvoir, c'est, plus souvent qu'autrement, un sentiment d'impuissance qui surgit, entre autres dans leurs rapports avec les femmes, même s'ill leur demeure caché comme ils se le cachent à eux-mêmes. Ce manque de pouvoir sur leur vie intérieure rend les hommes dépendants du soutien émotionnel des femmes. Il se sentent obligés de gagner l'amour, l'affection et l'accès à la sexualité par le biais de leurs performances et non par un partage réel d'intimité. Cette dépendance créerait aussi un sentiment d'impuissance. Ainsi, les femmes ne sont pas toujours conscientes du pouvoir qu'elles exercent sur les hommes. 
Farrell remet également en question l'idée, très répandue, que les femmes ont beaucoup plus évolué que les hommes par rapport aux rôles traditionnels. $\AA$ cette fin, il effectue une analyse très détaillée des revues et des romans populaires qui se vendent presque exclusivement aux femmes, y compris plusieurs nouvelles revues s'adressant aux "femmes de carrière ". II va jusqu'à redessiner, de façon parfois amusante, des annonces publicitaires et des bandes dessinées en inversant les rôles masculins et féminins afin d'en dévoiler les stéréotypes cachés. Selon cette analyse, un des fantasmes les plus puissants, chez les femmes, est encore d'épouser un homme "performant " sur le plan financier. À cause du contexte économique des années 1980, moins favorable aux femmes, Farrell croit que beaucoup d'entre elles continuent à chercher un compagnon pour obtenir leur sécurité matérielle. Malgré les changements apportés par le mouvement féministe, le message principal adressé aux hommes serait le suivant : "Soyez forts, réussissez dans la vie et vous aurez droit à l'amour et à la sexualité ".

Farrell admet que les femmes d'aujourd'hui veulent aussi des compagnons sensibles, capables d'exprimer leurs émotions. Cependant, il n'est pas convaincu qu'elles seraient toujours réceptives aux véritables sentiments des hommes : par exemple, comment ils peuvent se sentir piégés, encouragés par les femmes à explorer leur vulnérabilité, d'une part, alors qu'elles continuent, d'autre part, d'exiger d'eux d'être une source de sécurité avant tout. Ou comment les hommes vivent difficilement la fidélité sexuelle et comment elle représente pour eux un sacrifice important qui n'est pas toujours apprécié à sa juste valeur.

Farrell conclut donc que les rôles traditionnels sont encore très enracinés dans la société. Plutôt que de rejeter la responsabilité sur les hommes, comme cela a été la tendance depuis dix ou quinze ans, il croit qu'il sera dans l'intérêt des deux sexes de mieux comprendre leurs expériences respectives. Le féminisme a fait ressortir, et avec raison, l'injustice que représente l'exclusion des femmes du pouvoir économique et social. Mais il est également important qu'elles comprennent l'impuissance que les hommes vivent dans leurs rôles traditionnels et comment, selon eux, elles entretiennent aussi le statu quo.

Ce livre a le mérite d'énoncer, noir sur blanc, un grand nombre de situations ressenties par les hommes, mais qu'ils trouvent " taboues " ou difficiles à articuler au sein même de leur vie, dans le contexte actuel. Je dois cependant avouer que la lecture de cet ouvrage a créé chez moi un certain malaise. En voulant attirer l'attention sur certains aspects de la réalité qui sont souvent ignorés, Farrell pousse son analyse trop loin : ses propos ont certainement leur part de vérité mais me semblent parfois exagérés, surtout quand il parle du pouvoir que les femmes exercent sur les hommes.

De plus, sa conception du pouvoir confond les dimensions économique, politique, affective et sexuelle, et place ainsi les hommes et les femmes dans une situation d'égalité, presque par définition. Une telle conception du pouvoir est bien adaptée pour l'individu qui recherche un meilleur équilibre dans sa vie, mais elle empêche une vraie analyse de l'inégalité entre les hommes et les femmes. Cela n'est pas surprenant, cependant, compte tenu que la démarche de Farrell s'inspire de ses ateliers de croissance personnelle. 
Enfin, est-ce une analyse objective des rapports entre les hommes et les femmes que Farrell nous présente ici, ou ne veut-il pas plutôt, et de façon très consciente, montrer l'autre côté de la médaille, c'est-à-dire le vécu des hommes dans cette période de remise en question des rôles sexuels? Malgré cette limitation, ou peut-être grâce à elle, ce livre représente une lecture extrêmement stimulante pour toute personne qui veut réfléchir sur la condition masculine d'aujourd'hui.

Thomas ANTIL

Agent de recherche,

Direction de la planification et de l'évolution,

Ministère de la santé et des services sociaux.

Agir contre la violence, par Ginette LAROUCHE, Montréal, Les éditions de La Pleine Lune, 1987, 550 pages.

Agir contre la violence présente une option féministe à l'intervention auprès des femmes qui ont subi de la violence en milieu conjugal. Cette violence peut se rapporter à de multiples formes d'agressions : physiques, psychologiques, verbales ou sexuelles. Délaissant l'approche familiale, Ginette Larouche a choisi d'aider les femmes violentées à exploiter leurs capacités et leurs compétences afin qu'elles puissent assurer leur protection et augmenter leur qualité de vie en "se choisissant " d'abord elles-mêmes.

Au Québec, Ginette Larouche fait figure de pionnière en matière de violence conjugale, et il est évident que ce livre s'appuie sur une solide expérience de praticienne et de formatrice. Son souci constant d'allier la théorie à la pratique fait de cet ouvrage un outil de travail bien documenté, agréable à lire, foisonnant de techniques de soutien et d'exercices utilisables, soit en entrevue individuelle, soit en groupe. L'auteure n'en est d'ailleurs pas à ses premières armes sur le sujet. Au début du livre, l'historique du modèle proposé en fait foi. Mentionnons aussi que Ginette Larouche a décrit son modèle d'intervention dans le Guide d'intervention auprès des femmes violentées publié en 1985 par la Corporation professionnelle des travailleurs sociaux du Québec. Le présent ouvrage est cependant beaucoup plus étoffé.

Dans l'avant-propos et le premier chapitre, portant sur les notions de base, l'auteure situe bien la violence dans son contexte socio-politique. "La violence, écrit-elle, est la forme ultime de l'oppression d'un groupe par un autre ou d'un sexe par l'autre." L'analyse féministe qui sous-tend son intervention relie les difficultés des femmes aux racines sociales de l'inégalité des sexes. Cette lecture de la réalité ne confond pas les conséquences de la violence avec la personnalité de la victime. La dépression, par exemple, est identifiée comme l'une des conséquences du vécu de violence et non comme une caractéristique de la femme battue. 